\title{
Role of Bleomycin in Treatment of Cystic Hygroma
}

\author{
Saxena $S^{1}$, Hajela $S^{2}$ \\ ${ }^{1}$ Dr. Sunil Kumar Saxena, Assistant Professor, Department of Surgery, ${ }^{2}$ Dr. Shalini Hajela, Assistant Professor, Department \\ of Pediatrics, Both are affiliated to Bundelkhand Medical College, Sagar, MP, India
}

Address for correspondence: Dr.Sunil Kumar Saxena, Email: sunilsaxena599@yahoo.com

\begin{abstract}
Introduction: Cystic hygroma is a congenital multiloculated lymphatic lesion which most commonly occurs in cervicofacial region (posterior triangle of neck) and axilla. It is a benign cystic variety and the commonest form of lymphangioma. It contains watery fluid consistent with lymph. Bleomycin is a chemotherapeutic agent which acts by causing breaks in DNA. We used bleomycin, an intralesional sclerosing agent as an alternative to surgical excision. Methods: After written informed consent of the guardians of the patients, all the patients were given intralesional bleomycin injection at the dose of $0.2 \mathrm{mg} / \mathrm{kg}$ body weight. Under all aseptic precautions, the lymphatic fluid was aspirated completely and injection bleomycin was given from same site. This injection was repeated at an interval of 4 weeks. Post operatively compression dressing was done for $24 \mathrm{hrs}$. Results: Complete resolution of cystic hygroma was seen in 21(42\%) patients while partial response in 18 (36\%). No response, which is accumulation of lymphatic fluid in the cyst after repeated injection of bleomycin for 4 times, was noted in $11(22 \%)$ patients. Complications such as local irritation, redness at the injection site or fever occurred in 7 patients which were controlled with antibiotic and antiallergic therapy. Conclusion: Intralesional bleomycin can be used as first line of management in patients with large cystic hygroma, since it avoids invasive surgery and reduces risk of damage to vital organs. It is a safe, simple and effective method and can be done on day care basis.
\end{abstract}

Keywords: Asceptic Fibrosis, Bleomycin, Cystic Hygroma, Cystic Lymphangioma

\section{Introduction}

Cystic hygroma is a congenital malformation of the lymphatic system. Hygroma in Greek means watercontaining tumour. They are cystic variety of lymphangioma. Cystic hygroma occurs more frequently as compared to other types of lymphangioma, and may compose of single (unilocular) or multiple (multilocular) macrocystic lesions having scarce communication with normal lymphatic channels [1].

Lymphangiomas are usually classified as capillary, cavernous or cystic lymphangiomas. They may also be classified more conveniently, on the basis of size of the cysts contained, as microcystic, macrocystic and mixed lymphangiomas. Microcystic lymphangioma consists of cysts measuring less than $2 \mathrm{~cm}$ in size, whereas the size of cysts in case of macrocystic lymphangioma is more than $2 \mathrm{~cm}$. The mixed lymphangioma is characterized by cysts of variable sizes, i.e. some cysts are more than $2 \mathrm{~cm}$ in size and others are less than $2 \mathrm{~cm}$. The size of the lymphangioma is directly related to the degree of Manuscript

received: $7^{\text {st }}$ Feb 2014

Reviewed: $10^{\text {th }}$ Feb 2014

Author Corrected: $19^{\text {th }} \mathrm{Feb} 2014$

Accepted for Publication: $27^{\text {th }}$ Feb 2014 obstruction of lymphatico-venous drainage as evidenced by histologic appearance [2]. The common locations are cervico-facial regions (especially posterior cervical triangle), axilla, mediastinum, groin and below tongue; occasionally, these malformations occur in liver, spleen, kidney and intestine [3].

On clinical examination, these lesions appear soft, compressible, non-tender, transluminant and without any bruit. Ultrasound of the lesion usually features multicystic lesion with internal septations and no blood flow is detected on color doppler ultrasonography. Other modalities like CT scan and MRI can be employed to delineate the lesion, in a better way.

A CT scan demonstrates multicystic, homogeneous, noninvasive density with low attenuation. These modalities are usually helpful in ascertaining the extent of the lesion and their association with nerves and vessels and are particularly useful, when surgical management of the lesion is contemplated. These cysts may produce milky, serous, sero sanguinous or straw-coloured fluid, when aspirated with a wide-bore needle [4] 
Intralesional sclerotherapy has become an acceptable method of treatment for lymphangiomas in children. It involves the use of a sclerosing agent that causes irritation of the endothelial lining of the lymphangioma, which leads to inflammation, fibrosis and involution.

Other modes of treatment that have been employed with variable results include complete surgical resection, incision and drainage, aspirations, radiation therapy [5], laser excision, radio-frequency ablation and cauterization [4].

Complete surgical resection, which was considered ideal treatment, is impossible in many cases due to the nature of cystic hygroma, which has propensity to infiltrate tissue planes and encircle important neurovascular structures. Tumour recurrences and nerve injuries are common complications following surgery.

Previously reserved for treatment of unresectable lymphangiomas or in cases of tumour recurrence following surgery, intralesional sclerotherapy has gained popularity over recent years. Several studies using bleomycin and a newer agent OK-432 as sclerosants, have shown that this method of treatment produces favourable results compared to surgery [5].

We have used bleomycin for intralesional sclerosant therapy of cystic hygroma, and the primary reason for choosing to use bleomycin is because it was available while OK-432 is not yet available in India.

\section{Materials and methods}

This was a prospective review of 50 successive cases of lymphangioma. The procedures were conducted in the Surgery Department of Bundelkhand Medical College, Sagar between January 2010 and January 2012.The diagnosis of lymphangioma in these patients was made on the basis of clinical examination and imaging findings, mainly ultrasound (USG) and computed tomography (CT). Ultrasonographic assessment was performed on all patients. Patients' age, sex, body weight, symptoms, lesion location and lesion size were recorded.
Under the effect of general anaesthesia in some patients and under local anaesthetic in others, and with strict aseptic precautions, the cystic components of the tumour were aspirated with a hypodermic syringe and $23 \mathrm{G}$ needle using ultrasonographic guidance. While keeping the tip of aspiration needle within a cyst lumen, $0.2 \mathrm{mg}$ per $\mathrm{kg}$ body weight of bleomycin aqueous solution (1.5 $\mathrm{mg} / \mathrm{ml}$ water) was injected. When more than one cyst was aspirated, the calculated dose was divided by the number of cysts aspirated and the divided dose was injected into each cyst.

This procedure was repeated after 4 weeks if the cystic component persisted and measured at least $1 \mathrm{~cm}$ in diameter. The maximum cumulative dose of bleomycin allowed was $5 \mathrm{mg}$ per $\mathrm{kg}$ body weight. Patients were admitted for at least 24 hours following the procedure and monitored for possible immediate and delayed complications of the treatment. After discharge, patients were followed-up after 2 to 4 weeks when clinical assessment and measurements were made. If the lesion was still present, it was assessed with USG to see if further intralesional bleomycin was feasible.

A repeat procedure was done when there were cysts that measured at least $1 \mathrm{~cm}$ in diameter. This was the minimum cyst size where the radiologists felt confident to aspirate and maintain tip of the needle within the cyst lumen for the bleomycin injection. Follow-up and additional injections were given until the lesion completely resolved or intralesional bleomycin was no longer feasible (cysts measured less than $1 \mathrm{~cm}$ ).

The response was graded as complete resolution (total disappearance), good response (showing $>50 \%$ reduction in size) and poor response (showing $<50 \%$ reduction or no change in size). Recurrence was defined as reappearance of the tumour after complete resolution or increase in size after initial significant reduction in size. When bleomycin sclerotherapy was no longer required or no longer feasible, the follow up period was increased to 3 monthly, then 6 monthly until the size of the lesion was stable, and then yearly. Patients who showed poor response were offered surgery.

\section{Results}

During the $2 \mathrm{yr}$ study period, 50 patients were included in the study, with ages ranging from 6 years to 15 years. Out of 50 patients, 34 patients were male and 16 patients were female. All patients had a swelling. Other symptoms included pain, tenderness and skin erythema. The most common site of lesion was the neck, followed by face, axilla, chest, buttocks and thigh.

Lesions of the face were sited over the pre and post-auricular regions. Chest lesions were sited at the sub-clavicular region. The number of procedures per patient varied from once to 6 times, with a cumulative dose of 1 to 20 mg bleomycin. Total follow-up period from the time of 1 st injection ranged from 2 to 24 months. 
Table-1: Site of Lesion

\begin{tabular}{|l|l|l|}
\hline & Total & \% \\
\hline Neck & 28 & 56 \\
\hline Axilla & 5 & 10 \\
\hline Parotid & 4 & 8 \\
\hline Chest wall & 3 & 6 \\
\hline Buttock & 4 & 8 \\
\hline Abdomen \& Upper thigh & 6 & 12 \\
\hline
\end{tabular}

Complete resolution was seen in $42 \%$ ( 21/50) of lesions, $36 \%(18 / 50)$ had good response and the remaining $22 \%(11 / 50)$ had poor response or no response. The observations were recorded at the end of follow-up or at the time the patient was last seen in the clinic, the first patient who failed intralesional sclerotherapy had six injections and minimal reduction in size was achieved. . After six injections, there were no further cysts large enough for aspiration and injection.

Table 2: Response to treatment

\begin{tabular}{|l|l|l|}
\hline & Number & \% \\
\hline Complete resolution & 21 & 42 \\
\hline Good response & 18 & 36 \\
\hline Poor/No Response & 11 & 22 \\
\hline
\end{tabular}

Immediate complications reported include skin erythema at injection site, local swelling, mild tenderness and fever, which were controlled by oral antipyretics. However, no life- threatening complications such as respiratory obstruction and severe hypersensitivity reaction to bleomycin were observed. No patient developed excessive scarring as a result of the procedure.

Table 3: Complications

\begin{tabular}{|l|l|l|}
\hline & Number & \% \\
\hline Fever & 7 & 35 \\
\hline Local pain & 10 & 50 \\
\hline Erythema & 3 & 15 \\
\hline Hematoma and cellulitis & 0 & 0 \\
\hline
\end{tabular}

\section{Discussion}

Cystic hygroma is a cystic lymphatoid lesion, also called as cystic lymphangioma and macrocystic lymphatoid malformation. Cystic Hygroma manifests at birth in up to $65 \%$ and presents by the age of 2 years in $80-90 \%$ of cases. The incidence of lymphangioma is reported to be from 1.5-2.8 per 1000, and it has no predilection for either sex or any race [5].

The reported incidence of these tumors is quite variable ranging from 1 in 1000 to 1 in 16000 live births [6]. Embryologically, these lesions are believed to originate from sequestration of lymphatic tissue from lymphatic sacs, during the development of lymphatico-venous sacs. These sequestered tissues fail to communicate with remainder of the lymphatic or venous system. Later on, dilatation of the sequestered lymphatic tissues ensues, resulting in the cystic morphology of these lesions [2]. The common locations in $75 \%$ cases are cervico-facial regions (especially posterior cervical triangle), in $20 \%$ cases axilla and rest $5 \%$ being superior mediastinum, groin, retroperitoneum, pelvis, bones, scrotum and below tongue. Occasionally, these malformations occur in liver, spleen, kidney and intestine. Omental cyst in omentum and mesenteric cyst in the mesentery of intestine represents parallel lesions at these locations [3].

Lymphatic malformations can be congenital or acquired. Acquired lesions generally arise from obstruction of the lymphatic system due to trauma or infection. This might represent dormant cystic hygroma that can appear at any age, trauma being a coincident event [7].

Cystic hygroma may be associated with Turners Syndrome, Noonan Syndrome, Trisomies (Down's Syndrome \{Trisomy 21\}, Trisomy 18, Trisomy 13), cardiac anomalies, Fetal hydrops $[1,4]$.

The most common symptoms are swelling and cosmetic deformity. A large lesion in the neck can compress vital structures, cause respiratory obstruction, dysphagia and symptoms of nerve compression [5].

Available online at: www.ijmrr.in 346 | P a g e 
The main indications of treatment are respiratory distress, recurrent infections, cosmetic reasons and compression of local structures like airway, blood vessel and upper gastrointestinal tract.

Intralesional sclerotherapy has become the preferred modality of treating cystic hygroma with increasingly documented remarkable results for management of such lesions with sclerosant agents although complete surgical excision used to be the ideal treatment in the presclerotherapy times. The other treatment modalities that have been employed with variable results include simple drainage, aspirations, radiation, laser excision, radiofrequency ablation and cauterization [8].

The idea of using sclerotherapy in the treatment of lymphangioma occurred when it was noted that lymphatic malformations spontaneously involute when they became infected and the infection resolved. The first case of lymphangioma treated by sclerotherapy was reported in 1933, using sodium morrhuate [5]. Complete tumour regression was noted in 6 weeks following intralesional injection [8]. Other sclerosing agents that have been used previously, namely iodine, ethanolamine oleate, alcohol, ethibloc, sodium tetradecyl sulfate, tetracycline and cyclophosphamide [5]. Bleomycin and OK-432(Picibanil) are currently the most popular sclerosants. However, only bleomycin is available in India.

Bleomycin is an anti-neoplastic antibiotic, produced by the fermentation of streptomyces verticillus. Discovered in 1965 by Umezawa, this drug was found to cause single strand and double strand DNA breaks and inhibition of DNA and RNA synthesis. Initially, when this antineoplastic drug was used in the treatment of malignant pleural effusion, it was observed that bleomycin caused marked fibrosis and scarring. This sclerosing property was first put to use in 1977 by Yura in the treatment of cystic hygroma [4]. Studies using bleomycin have produced promising results in cases of cystic hygroma [5].

Side effects of Bleomycin are fever, transient increase in size of swelling, hemorrhage, leukocytosis, infection, and pulmonary fibrosis. The primary concern of bleomycin therapy is its risk of pulmonary toxicity. The risk is dose related with an increased incidence associated with a total dose exceeding $30 \mathrm{mg} / \mathrm{m}^{2}$ of body surface area or total cumulative dose of $400 \mathrm{mg}$ given intra-venously to oncology patients. Elderly oncology patients and those with underlying pulmonary disease and renal failure were at greater risk [5]. Bleomycin doses used in sclerotherapy are small in comparison, typically $1 \%$ to $5 \%$ of the lowest dose associated with possible pulmonary fibrosis. [9] We used $0.2 \mathrm{mg} / \mathrm{kg}$ of dose. Others have used it in doses ranging from 0.3 to $3 \mathrm{mg} / \mathrm{kg}$. [10].

In our study, after intralesional bleomycin therapy, complete resolution was seen in $42 \%(21 / 50)$ of lesions, $36 \%(18 / 50)$ had good response and the remaining $22 \%$ $(11 / 50)$ had poor response or no response. Our study indicated that intralesional bleomycin therapy was very effective and the results were comparable to various published series. Other authors have also quoted success rates of between 36 to $63 \%$ for complete tumour regression, of up to $88 \%$ significant lesion regression, and poor response of between 12 to $23 \%$ using either bleomycin or OK-432. Rozman et al noted excellent and good response in $63 \%(15 / 24)$ of lesions and $21 \%(5 / 24)$ patients [5]. Niramis et al noted $83 \%$ of response [11] where as Baskin et al noted about $95 \%$ of response [12], suggesting good activity of bleomycin for the purpose. Okada observed $86 \%$ (25cases) showed significant reduction of the mass and 55\% (16 cases) showed complete disappearance in a study on intralesional sclerotherapy in 29 cases [8]. Orford observed an excellent (complete clinical resolution) response in seven (44\%) patients, a good ( $>50 \%$ response) result in seven (44\%), and a poor or no response in two (12\%) suggesting that bleomycin intralesional sclerosant is effective therapy for cystic hygroma, with response rates comparable to those of surgical removal, but with the advantage of avoiding inadvertent nerve damage and scarring [10].

No serious complications were seen as a result of intralesional bleomycin therapy. Most patients complained of mild skin erythema, local swelling, induration, mild tenderness and fever. These symptoms lasted for a day or two but did not prolong their stay in hospital. In this study, few complications were noted as also noted by Rozmann [5], whereas Niramis et al noted complications in about $43 \%$ of patients [11]. Clinical studies done at various places in which patients were treated with intralesional bleomycin did not report pulmonary fibrosis as a complication.

Although surgical excision has been considered to be treatment of choice by most of the surgeons, it is associated with tedious dissection along with lot of morbidity in the form of disfigurement and damage to vital structures adjacent to the mass including damage to a neurovascular structure (including cranial nerves), chylous fistula, chylothorax, hemorrhage, infection, wound seroma, ugly scar and recurrence. Recurrence 
rates vary depending on the complexity of the lesion and the completeness of excision. If residual tissue is left behind, the expected recurrence rate is approximately $15 \%$. Most recurrences occur within the first year but have been reported to occur as long as 10 years after excision. Postoperative complications occur in $30 \%$ or more of cases.

Therefore, sclerotherapy of lymphangioma has gained popularity during recent years. In this prospective study, we found the response satisfactory in almost $78 \%$ of cases (Complete resolution in $42 \%$ and Good response in $36 \%$ ) as also observed in various case series. In short term follow up no recurrence was encountered. Others have also not found recurrence to be of much concern [5, 10]. Although mortality has been reported by Niramis et al [11], it was not seen in this series.

\section{Conclusion}

Intralesional bleomycin therapy is a safe and effective method of treatment for lymphangioma. The results from our series are comparable with other published series and support the continued use of sclerotherapy with bleomycin in the treatment of lymphangioma. No serious side effects from intralesional bleomycin therapy were observed. Sclerotherapy is recommended in place of surgery as the first-line treatment modality. The latter must be reserved for those lesions where sclerotherapy had failed. However continued trials and long term follow up is required to establish intralesional sclerotherapy with bleomycin as the gold standard for the treatement of cystic hygroma.

Funding: Nil

Conflict of Interest: Nil.

\section{References}

1. Manikoth P, Mangalore G P, Megha V. Axillary Cystic Hygroma. J Postgrad Med 2004;50:215-6

2. Fonkalsrud EW. Lymphatic disorders. In: Grosfeld JL, O'Neill JA Jr, Coran JA, Fonkalsrud EW, Caldamone AA, editors. Pediatric surgery. 6th ed. Chicago: Mosby Elsevier; 2006. pp. 2137-45.
3. Robbins SL, Cotran RS, Kumar V. Blood vessels. In: Robbins SL, Cotran RS, Kumar V, editors. Pathologic barir of dir Philadelphia: WB Saunders Co; 1984, pp 544.

4. Bilal Mirza, Lubna Ijaz, Muhammad Saleem, Muhammad Sharif, and Afzal Sheikh. Cystic Hygroma: An Overview.J Cutan Aesthet Surg. 2010 Sep-Dec; 3(3): 139-144.

5. Rozman Z, Thambidorai RR, ZalehaAM, ZakariaZ, ZulfiqarMA. Lymphangioma: Is intralesional bleomycin sclerotherapy effective? Biomed Imaging Interv J. 2011 Jul-Sep; 7(3): e18.

6. Brooks JE. Cystic hygroma of neck. Laryngoscope. 1973;83:117-28.

7. Brock ME, Smith RJ, Parey SE, Mobley DL. Lymphangioma. An otolaryngologic perspective. Int J Pediatr Otorhinolaryngol. 1987;14:133-40.bMed]

8. Okada A, Kubota A, Fukuzawa M, Imura K, Kamata S. Injection of Bleomycin as a Primary Therapy of Cystic Lymphangioma, J Pediatr Surg 1992; 27(4): 440-443.

9. Mahajan JK, Bharathi V, Chowdhary SK, Samujh R, Menon P, Rao KL. Bleomycin as intralesional sclerosant for cystic hygromas. J Indian Assoc Pediatr Surg 2004;9:3-7.

10. Kumar V, Kumar P, Pandey A, Gupta DK, Shukla RC, Sharma SP, and Gangopadhyay AN. Intralesional Bleomycin in Lymphangioma: An Effective and Safe Non-Operative Modality of Treatment. J Cutan Aesthet Surg. 2012 Apr-Jun; 5(2): 133-136.

11. Niramis $R$, Watanatittan $S$, Rattanasuwan $T$. Treatment of cystic hygroma by intralesional bleomycin injection: experience in 70 patients. Eur $J$ Pediatr Surg. 2010 May;20(3):178-82.

12. Baskin D, Tander B, Bankaoglu M. Local bleomycin injection in the treatment of lymphangioma. Eur $J$ Pediatr Surg 2005;15:383-6.

How to cite this article?

Saxena S, Hajela S. Role of Bleomycin in Treatment of Cystic Hygroma. Int J Med Res Rev 2014;2(4):344- 348. doi:10.17511/ijmrr.2014.i04.16 